\title{
STRUCTURE OF CORADICAL FILTRATION AND ITS APPLICATION TO HOPF ALGEBRAS OF DIMENSION $p q$
}

\author{
DAIJIRO FUKUDA \\ e-mail:d-fukuda@world.odn.ne.jp
}

(Received 16 October, 2007; revised 19 October, 2007; accepted 28 October, 2007)

\begin{abstract}
This paper contributes to the classification problem of $p q$ dimensional Hopf algebras $H$ over an algebraically closed field $\mathbf{k}$ of characteristic 0 , where $p, q$ are odd primes. It is shown that such Hopf algebras $H$ are semisimple for the pairs of odd primes $(p, q)=(3,11),(3,13),(3,19),(5,17),(5,19),(5,23),(5,29),(7,17),(7,19),(7,23)$, $(7,29),(11,29),(13,29)$.
\end{abstract}

2000 Mathematics Subject Classification. 16W30.

1. Introduction. From the beginning of the 1990s, the classification of finite dimensional Hopf algebras over an algebraically closed field was actively studied, and remarkable developments were accomplished. Especially, for a Hopf algebra $H$ of dimension $p q$ over an algebraically closed field of characteristic 0 , P. Etingof and $\mathrm{S}$. Gelaki showed that if $H$ is semisimple then it is isomorphic to a group algebra or the dual of a group algebra [4]. And D. Ştefan showed that if $H$ is pointed then it is semisimple [10]. However the classification of general non-semisimple, non-pointed Hopf algebras of dimension $p q$ is still open.

The following results are also obtained about the classification problem in the dimension $p q$. S.-H. Ng showed that a Hopf algebra $H$ of dimension $p^{2}$ is either semisimple or isomorphic to a Taft algebra [7]. N. Andruskiewitsch and S. Natale solved problems for 15,21, 25, 35 and 49 dimensions using a decomposition of the first term $H_{1}$ of the coradical filtration [1]. M. Beattie and S. Dăscălescu solved problems for $14,55,77,65,91$ and 143 dimensions by examining the first term of the coradical filtration more closely [2]. Recently S.-H. $\mathrm{Ng}$ solved the case where $p, q$ are twin primes in [8], and the case for dimension $2 p$ in [9]. Furthermore P. Etingof and S. Gelaki solved the case where $q \leq 2 p+1$ [5]. For distinct prime numbers $p, q$, all the $p q$ dimensional examples known so far are semisimple. So it is natural to ask whether that all $p q$ dimensional Hopf algebras are semisimple.

In this paper we apply the technique of the decomposition of the first term $H_{1}$ of the coradical filtration used in [1] and [2] to general $n$-th term $H_{n}$ of the coradical filtration, and contribute to the question above by proving the following main Theorem.

THEOREM 1.1. Let $H$ be a pq dimensional Hopf algebra over an algebraically closed field of characteristic 0 where $p$, q are odd primes with $p<q<4 p+10, q \leq 29$ such that $q$ can not be expressed as $q=4 p+2+p z_{1}+4 z_{2}$ with positive integers $z_{1}, z_{2}$. Then $H$ is semisimple.

As a corollary to Theorem 1.1, our new classification results are the following. 
Corollary 1.2. A Hopf algebra of dimension pq where $(p, q)=(3,11),(3,13)$, $(3,19),(5,17),(5,19),(5,23),(5,29),(7,17),(7,19),(7,23),(7,29),(11,29),(13,29)$ is semisimple and isomorphic to a group algebra or the dual of a group algebra.

2. Preliminaries. Throughout this paper, $H$ is a finite dimensional Hopf algebra over an algebraically closed field $\mathbf{k}$ of characteristic 0 , and $\Delta, \epsilon, S$ denote the comultiplication, the counit and the antipode respectively. The $n$-th term of the coradical filtration of $H$ is $H_{n}=\wedge^{n+1} H_{0}$, where $H_{0}$ is the coradical of $H$. As $\mathbf{k}$ is algebraically closed, there exists a coalgebra projection $\pi: H \rightarrow H_{0}$ and $H=H_{0} \oplus I$, where ker $\pi=I$ (see [6, 5.4.2]). Refer to [3] for general results of Hopf algebras.

Set $\rho_{l}=(\pi \otimes i d) \Delta$ and $\rho_{r}=(i d \otimes \pi) \Delta . H$ is a $H_{0}$-bicomodule with the structure maps $\rho_{l}$ and $\rho_{r} . H_{0}, H_{n}, I$ are $H_{0}$-subbicomodules of $H$. Any $H_{0}$-bicomodule is a direct sum of simple $H_{0}$-subbicomodules and a simple $H_{0}$-bicomodule has coefficient coalgebras $\left(C_{i}, C_{j}\right)$ and its dimension is $n_{i} n_{j}$, where $n_{i}, n_{j}$ are the dimensions of associated comodules of $C_{i}, C_{j}$ respectively.

Let $P_{n}, n=0,1,2, \ldots$ be defined inductively by:

$$
\begin{gathered}
P_{0}=0, \\
P_{1}=\left\{x \in H ; \Delta(x)-\rho_{l}(x)-\rho_{r}(x)=0\right\}, \\
P_{n}=\left\{x \in H ; \Delta(x)-\rho_{l}(x)-\rho_{r}(x) \in \sum_{1 \leq i \leq n-1} P_{i} \otimes P_{n-i}\right\}, n \geq 2 .
\end{gathered}
$$

Then $P_{n}$ is a $H_{0}$-subbicomodule of $I$ and $P_{n}=H_{n} \cap I$, due to Nichols (see [1, Lemma 1.1]).

We denote by $P_{n}^{C_{i}, C_{j}}$ the isotypic component of the simple subbicomodule of $P_{n}$ with coalgebra of coefficients $\left(C_{i}, C_{j}\right)$. We say the subspace $P_{n}^{C_{i}, C_{j}}$ is non-degenerate if $P_{n}^{C_{i}, C_{j}} \not \subset P_{n-1}$.

We list several key results which we use in this paper for the readers convenience.

Proposition 2.1 ([Lemma 1.2, 1]). The first term of the coradical filtration can be expressed as $C_{1}=\sum_{i, j} C_{i} \wedge C_{j}$ and $C_{i} \wedge C_{j}=C_{i} \oplus C_{j} \oplus P_{1}^{C_{i}, C_{j}}$.

Proposition 2.2 ([Crollary 1.3, 1]). For a grouplike element $g$ and the antipode $S$,

$$
\operatorname{dim} P_{1}^{C, D}=\operatorname{dim} P_{1}^{g C, g D}=\operatorname{dim} P_{1}^{C g, D g}=\operatorname{dim} P_{1}^{S(D), S(C)} .
$$

The following results are from [2].

Proposition 2.3 ([Crollary 4.2, 2]). If there is no non-trivial skew primitives then there exists a simple subcoalgebra $C(\operatorname{dim} C \geq 4)$ of $H$ such that $P_{1}^{1, C} \neq 0$.

Proposition 2.4 ([Lemma 5.1, 2]). No non-semisimple Hopf algebra H of squarefree dimension can be generated as a Hopf algebra by a simple subcoalgebra of dimension 4 that is stable under the antipode.

In general the orders of the grouplike elements and the antipode are important factors for discussion of finite dimensional Hopf algebras. The following result gives this for non-semisimple Hopf algebras of dimension $p q$ where $p, q(p<q)$ are odd primes.

Proposition 2.5 ([Proposition 6.2, 7]). If H is a non-semisimple Hopf algebra of dimension pq then the order of any grouplike element is 1 or $p$ and the order of the antipode is $4 p$. 
3. Some properties of coradical filtration. In this section we will obtain some properties of the space $P_{n}$. Throughout this section, $C, D, Z, W$ and $X_{1}, \ldots, X_{i}, \ldots$ are simple subcoalgebras of $H$. We denote $\Delta-\rho_{l}-\rho_{r}$ by $\widehat{\Delta}$. Then the kernel of $\widehat{\Delta}$ is $P_{1}$. $\widehat{\Delta}$ has a coassociativity inherited from the coassociativity of $\Delta$.

Suppose $\widehat{\Delta}^{(n)}(x)=0$ for $x \in H$. Setting a base of $P_{k}$ by extending the base of $P_{k-1}$ inductively, $\widehat{\Delta}^{(m)}(x)$ is contained in $\sum_{\substack{1 \leq i_{j} \leq n-1 \\ i_{1}+\cdots+i_{m+1}=n}} P_{i_{1}} \otimes \cdots \otimes P_{i_{m+1}}$ by induction on $m$. Thus $x$ is contained in $P_{n}$, and so the space $P_{n}$ can be expressed as $\left\{x \in H \mid \widehat{\Delta}^{(n)}(x)=0\right\}$.

Lemma 3.1. If $x \in P_{n}, x \notin P_{n-1}$ then $\widehat{\Delta}(x) \notin \sum_{\substack{1 \leq i \leq n-1 \\ i \neq m}} P_{i} \otimes P_{n-i}$ for all $m \in$ $\{1, \ldots, n-1\}$.

Proof. We suppose that $\widehat{\Delta}(x)$ is contained in $\sum_{\substack{1 \leq i \leq n-1 \\ i \neq m}} P_{i} \otimes P_{n-i}$ for some $m \in\{1, \ldots, n-1\}$. Thus $\widehat{\Delta}^{(n-1)}(x)=\left(\widehat{\Delta}^{(m-1)} \otimes \widehat{\Delta}^{(n-m-1)}\right) \widehat{\Delta}(x)=0$. This means $x \in$ $P_{n-1}$.

LEMMA 3.2. If the subspace $P_{n}^{C, D}$ is non-degenerate then there exists a set of simple coalgebras $\left\{X_{1}, \ldots, X_{n-1}\right\}$ such that $P_{i}^{C,}, X_{i}, P_{n-i}^{X_{i}, D}$ are non-degenerate for all $i \in\{1, \ldots$, $n-1\}$.

Proof. Since $\Delta\left(P_{n}^{C, D}\right) \subset C \otimes P_{n}^{C, D}+P_{n}^{C, D} \otimes D+\sum_{\substack{1 \leq i \leq n-1 \\ j, k, l, m}}\left(P_{i}^{X_{j}, X_{k}} \otimes P_{n-i}^{X_{l}, X_{m}}\right)$, and using the coassociativity, $\Delta^{(2)}\left(P_{n}^{C, D}\right)$ is contained in the following space.

$$
\begin{aligned}
& C \otimes C \otimes P_{n}+C \otimes P_{n} \otimes D+P_{n} \otimes D \otimes D+I \otimes I \otimes I \\
& \quad+\sum_{i, j, k, l, m}\left(C \cap X_{j}\right) \otimes P_{i}^{X_{j}, X_{k}} \otimes I+P_{i}^{X_{j}, X_{k}} \otimes\left(X_{k} \cap X_{l}\right) \otimes P_{n-i}^{X_{l}, X_{m}} \\
& \quad+I \otimes P_{n-i}^{X_{l}, X_{m}} \otimes\left(D \cap X_{m}\right) .
\end{aligned}
$$

Thus the following result can be obtained by applying the counit $\epsilon$.

$$
\Delta\left(P_{n}^{C, D}\right) \subset C \otimes P_{n}+P_{n} \otimes D+\sum_{i, k} P_{i}^{C,} X_{i}^{(k)} \otimes P_{n-i}^{X_{i}^{(k)}}, D .
$$

If it lacks even one of the simple coalgebras $X_{1}, \ldots, X_{n-1}$ such that $P_{i}^{C, X_{i}}, P_{n-i}^{X_{i}, D}$ are non-degenerate, then $P_{n}^{C, D}$ is contained in $P_{n-1}$ by Lemma 3.1. This contradicts non-degeneracy of $P_{n}^{C, D}$.

Lemma 3.3. The space $C \wedge\left(\wedge^{n-1} H_{0}\right) \wedge D$ which is defined by the wedge product of simple coalgebras can be decomposed as follows.

$$
C \wedge\left(\wedge^{n-1} H_{0}\right) \wedge D= \begin{cases}C \oplus D \oplus P_{1}^{C, D} & \text { for } n=1 \\ H_{0} \oplus\left(P_{n-2}+\sum_{i, j}\left(P_{n-1}^{C, X_{i}}+P_{n-1}^{X_{j}, D}\right)+P_{n}^{C, D}\right) & \text { for } n \geq 2\end{cases}
$$

Proof. N. Andruskiewitsch, S. Natale proved the case $n=1$ (see Proposition 2.1). We suppose $n \geq 2$.

We first show that the left-hand side is contained in the right-hand side by induction on $n$. We assume $P_{n}^{Z, W}$ is non-degenerate and $x \in P_{n}^{Z, W} \cap\left(C \wedge\left(\wedge^{n-1} H_{0}\right) \wedge D\right)$. By Lemma 3.2, there exist a set of simple coalgebras $\left\{X_{1}^{(k)}, \ldots, X_{n-1}^{(k)}\right\}$ such that $\Delta(x)$ is contained in the intersection of the following two spaces

$$
\begin{gathered}
C \otimes H+H \otimes\left(\wedge^{n-1} H_{0} \wedge D\right), \\
Z \otimes P_{n}^{Z, W}+P_{n}^{Z, W} \otimes W+\sum_{i, k} P_{i}^{Z, X_{i}^{(k)}} \otimes P_{n-i}^{X_{i}^{(k)}, W} .
\end{gathered}
$$


Thus $\Delta(x) \in(Z \cap C) \otimes P_{n}^{Z, W}+Z \otimes\left(P_{n-2}+P_{n-1}^{Z,(W \cap D)}\right)+P_{n}^{Z, W} \otimes W+I \otimes I$. If $Z \neq C$ then $\Delta(x)$ is contained in $Z \otimes P_{n-1}+I \otimes W+I \otimes I$. Therefore $x=(\epsilon \otimes$ id $) \Delta(x) \in P_{n-1}$ and so $C \wedge\left(\wedge^{n-1} H_{0}\right) \wedge D$ is contained in $H_{0} \oplus\left(P_{n-1}+\sum_{i} P_{n}^{C,} X_{i}\right)$. We can show that $C \wedge\left(\wedge^{n-1} H_{0}\right) \wedge D \subset H_{0} \oplus\left(P_{n-1}+\sum_{i} P_{n}^{X_{i}, D}\right)$ in the same way. Moreover $P_{n-1} \cap\left(C \wedge\left(\wedge^{n-1} H_{0}\right) \wedge D\right)$ is contained in $P_{n-2} \oplus \sum_{i j} P_{n-1}^{C, X_{i}}+P_{n-1}^{X_{j}, D}$ similarly. Therefore the left-hand side is contained in the right-hand side.

We next show that the right-hand side is contained in the left-hand side. By the definition of wedge and the inductive argument, the spaces $P_{n-1}^{C, X}, P_{n-1}^{X, D}, P_{n}^{C, D}$ are contained in $C \wedge\left(\wedge^{n-1} H_{0}\right) \wedge D$ for all simple coalgebras $X$. Hence the right-hand side is contained in the left-hand side.

COROLlary 3.4. The space $C \wedge\left(\wedge^{n-1} H_{0}\right) \wedge D$ can be decomposed as following,

$$
H_{0} \oplus\left(\bigoplus_{X \neq C, Y \neq D} P_{n-2}^{X, Y}\right) \oplus\left(\bigoplus_{X \neq D} P_{n-1}^{C, X}\right) \oplus\left(\bigoplus_{X \neq C} P_{n-1}^{X, D}\right) \oplus P_{n}^{C, D} \quad \text { for } n \geq 2 .
$$

LEMMA 3.5. For a grouplike element $g$ and the antipode $S$,

$$
\operatorname{dim} P_{n}^{C, D}=\operatorname{dim} P_{n}^{g C, g D}=\operatorname{dim} P_{n}^{C g, D g}=\operatorname{dim} P_{n}^{S(D), S(C)} .
$$

Proof. Since $g\left(C \wedge H_{0} \wedge \cdots \wedge H_{0} \wedge D\right)=g C \wedge H_{0} \wedge \cdots \wedge H_{0} \wedge g D$, the first equality is obtained by the counting dimensions of $g C \wedge H_{0} \wedge \cdots \wedge H_{0} \wedge g D$ and $C \wedge H_{0} \wedge \cdots \wedge H_{0} \wedge D$ with Corollary 3.4. The rest can be shown similarly.

This Lemma is a generalization of Proposition 2.2 which deals with the case $n=1$. And the proof is based on the proof of Proposition 2.2(see [1, Corollary 1.4]).

Let the socle of $H$ be $\operatorname{Soc}(H)=\oplus_{i, j} M_{C_{i}}^{j}$ where $M_{C_{i}}^{1}, \ldots, M_{C_{i}}^{k}$ are all simple subcomodules of $C_{i}$ as a right $H$-comodule. Let $E\left(M_{C_{i}}^{j}\right)$ be an injective envelope of $M_{C_{i}}^{j}$. Then $H$ can be expressed as $H=\oplus_{i, j} E\left(M_{C_{i}}^{j}\right)$ as a right $H$-comodule [3, Theorem 2.4.16]. To simplify the description, we denote the sum of above injective envelopes $\oplus_{j} E\left(M_{C_{i}}^{j}\right)$ by $E\left(C_{i}\right)$ for fixed $C_{i}$.

LEMMA 3.6. The space $E(C)$ can be expressed as $C \oplus \sum_{n, D} P_{n}^{C, D}$.

Proof. We first show that the left-hand side is contained in the right-hand side. Since $H=H_{0} \oplus I=H_{0} \oplus \sum_{n, X, Y} P_{n}^{X, Y}$, it is clear that $E(C) \subset C \oplus \sum_{n, X, Y} P_{n}^{X, Y}$. Thus it is sufficient to show $X=C$. We consider $E(C) \cap P_{n}^{X}, Y$ under the assumption $X \neq C$.

$$
\begin{aligned}
E(C) \cap P_{n}^{X, Y} & =(\epsilon \otimes i d) \Delta\left(E(C) \cap P_{n}^{X, Y}\right) \\
& \subset(\epsilon \otimes i d)\left((E(C) \otimes H) \cap\left(X \otimes P_{n}+P_{n} \otimes Y+I \otimes I\right)\right) \\
& \subset(\epsilon \otimes i d)(I \otimes H)=0 .
\end{aligned}
$$

Next we show that the right-hand side is contained in the left-hand side. Let $M_{D}$ be a simple subcomodule of the simple coalgebra $D$ which is not equal to $C$. If $E\left(M_{D}\right) \cap$ $\left(C \oplus \sum_{n, X} P_{n}^{C, X}\right) \neq 0$ then such intersection is a subcomodule of $E\left(M_{D}\right)$ and it contains $M_{D}$, contradicting $D \neq C$. Therefore $E\left(M_{D}\right) \cap\left(C \oplus \sum_{n, X} P_{n}^{C, X}\right)=0$.

LEMMA 3.7. Let $C, D$ be simple subcoalgebras of $H$ such that $P_{m}^{C, D}$ is non-degenerate. If $\widehat{\Delta}\left(P_{n}^{C, X}\right) \subset \sum_{\substack{1 \leq i \leq n-1 \\ Y \neq D}}\left(P_{i}^{C, Y} \otimes P_{n-i}^{Y, X}\right)+P_{m-1}^{C, D} \otimes P_{n-m+1}^{D, X}$ for all simple coalgebras $X$ 
and all $n \in \mathrm{N}$. Then Head $(E(C))=E(C) / \mathbf{J} E(C)$ contains D-simple comodules as a direct summand where $\mathbf{J}=H_{0}^{\perp}$ is the Jacobson radical of $H^{*}$.

Proof. Set $\Phi=C \oplus\left(\sum_{n, Y \neq D} P_{n}^{C, Y}\right) \oplus P_{m-1}^{C, D}$. The space $\Phi$ is a subcomodule of $E(C)$ by the condition above.

Since $\widehat{\Delta}(E(C)) \subset \sum_{\substack{n, X, Y \\ Y \neq D}}\left(P_{n}^{C, Y} \otimes P_{n}^{Y, X}\right)+P_{m-1}^{C, D} \otimes P_{n}^{D, X}$, the following holds.

$$
\begin{aligned}
J E(C) & =\sum_{\substack{n, X, Y \\
Y \neq D}}\left\langle E(C)_{(2)}, J\right\rangle E(C)_{(1)} \\
& \subset \sum_{\substack{\text { in }\\
}}\left(\left\langle H_{0}, H_{0}^{\perp}\right\rangle I+\left\langle I, H_{0}^{\perp}\right\rangle C+\left\langle P_{n}^{Y, X}, H_{0}^{\perp}\right\rangle P_{n}^{C, Y}+\left\langle P_{n}^{D, X}, H_{0}^{\perp}\right\rangle P_{m-1}^{C, D}\right) \\
& \left.P_{n}^{C, Y}\right) \oplus P_{m-1}^{C, D}=\Phi .
\end{aligned}
$$

Thus there exists a natural projection $E(C) / \mathbf{J} E(C)$ to $E(C) / \Phi$.

Since $E(C) / \mathbf{J} E(C)$ and $E(C) / \Phi$ are semisimple and $E(C) / \Phi$ contains $D$-simple comodules as a direct summand, $E(C) / \mathbf{J} E(C)$ also contains $D$-simple comodules as a direct summand.

LEMMA 3.8. Let $C, D$ be simple subcoalgebras such that $P_{m}^{C, D}$ is non-degenerate. If $\operatorname{dim} C \neq \operatorname{dim} D$ or $\operatorname{dim} P_{m}^{C, D}-\operatorname{dim} P_{m-1}^{C, D} \neq \operatorname{dim} C$ then there exists a simple subcoalgebra E such that $P_{l}^{C, E}$ is non-degenerate for some $l \geq m+1$.

Proof. We assume that there is no simple subcoalgebra $E$ such that $P_{l}^{C, E},(l \geq m+$ $1)$ is non-degenerate. Since the simple coalgebra $D$ satisfies the condition in Lemma 3.7, $\operatorname{Head}(E(C))$ contains $D$-simple comodules as a direct summand. On the other hand $\operatorname{Head}(E(C))=\operatorname{Soc}(E(\pi(C)))=\pi(C)$ where $\pi$ is a permutation on the set of all simple coalgebras of $H$, since $H$ is coFrobenius. Then $\pi(C)=D$ and thus $\operatorname{dim} C=\operatorname{dim} D$.

Moreover, if $\operatorname{dim} C=\operatorname{dim} D$ and $\operatorname{dim} P_{m}^{C, D}-\operatorname{dim} P_{m-1}^{C, D} \neq \operatorname{dim} C$ then the following holds where $\Phi$ is the subspace in the proof of Lemma 3.7.

$$
\begin{aligned}
& \operatorname{dim} C<\operatorname{dim} P_{m}^{C, D}-\operatorname{dim} P_{m-1}^{C, D}=\operatorname{dim} P_{m}^{C, D} / P_{m-1}^{C, D}=\operatorname{dim} E(C) / \Phi \\
& \quad \leq \operatorname{dim} \operatorname{Head}(E(C))=\operatorname{dim} C .
\end{aligned}
$$

4. Proof of Theorem 1.1. In this section we will show Theorem 1.1 using Lemmas 3.2, 3.5 and 3.8. Let $H$ be a non-semisimple Hopf algebra of dimension $p q$ where $p, q$ are odd primes such that $p<q<4 p+10, q \leq 29$. We can assume the order of the antipode is $4 p$ and $G(H)$ is isomorphic to the cyclic group $\mathbf{C}_{p}$ by Proposition 2.5, and there exists a simple coalgebra $C$ such that $P_{1}^{1, C} \neq 0, \operatorname{dim} C \geq 4$ by Proposition 2.3. Let $\operatorname{dim} C=m^{2}$, and set $C_{1}=C, C_{2}=g C, \ldots, C_{p}=g^{p-1} C$ where $g \neq 1_{H} \in G(H)$. If $(m, p)=1$ then $C_{i} \neq C_{j}$ for $i \neq j$.

LEMMA 4.1. If $P_{1}^{1, C_{1}} \neq 0, \operatorname{dim} C_{1}=4$ then there exists $k \in\{1, \ldots, p\}$ such that $S\left(C_{k}\right) \notin\left\{C_{1}, \ldots, C_{p}\right\}$.

Proof. The proof is by contradiction. Suppose that the antipode $S$ induces a permutation $\sigma$ on the set $\left\{C_{1}, \ldots, C_{p}\right\}$. Each $C_{i}$ is not stable under the antipode $S$ from Proposition 2.4, hence the permutation $\sigma$ has no fixed points. The order of $\sigma$ is a divisor of $4 p$, so any cycle has length 2 or 4 or $p$. However, if there exists a cycle with length 
2 or 4 then $\sigma$ has fixed points since $p$ is an odd prime. Thus $\sigma$ has a cycle of length $p$. Then $P_{1}^{1, C_{1}} \neq 0$ implies that there exist $2 p$ disjoint $P_{1}^{S\left(C_{1}\right), 1}, P_{1}^{1, S^{2}\left(C_{1}\right)}, \ldots, P_{1}^{S^{p-1}\left(C_{1}\right), 1}$ by using Lemma 3.5 , and so $P_{1}$ contains $2 p^{2}$ disjoint subspaces $P_{1}^{g^{l}, C_{j}}, P_{1}^{C_{i}, g^{j}}$, using Lemma 3.5 again. The dimension of each $P_{1}^{g^{i}, C_{j}}$ is greater than or equal to 2 , hence $\operatorname{dim} P_{1} \geq 4 p^{2}$. Furthermore, by Lemma 3.8, there exist 4 dimensional simple subcoalgebras $\left\{\bar{C}_{i}\right\}$ and grouplike elements $\left\{g^{i}\right\}$ such that $P_{n}^{C_{i}}, \bar{C}_{j}, P_{n^{\prime}}^{g^{i}, g^{j}}\left(n, n^{\prime} \geq 2\right)$ are non-degenerate. Thus $\operatorname{dim} I-\operatorname{dim} P_{1} \geq p+4 p$ and so $\operatorname{dim} H \geq(p+4 p)+4 p^{2}+(p+4 p)=4 p^{2}+10 p$. This contradicts the condition $q<4 p+10$.

We denote ${ }^{X} I^{Y}$ the total sum of the subspaces of $I$ whose existence is induced from the fact $P_{1}^{X, Y} \neq 0$ by repeated application of Lemmas 3.2, 3.5 and 3.8, as in the second half of the proof of Lemma 4.1.

Let $C_{1}$ be a simple subcoalgebra such that $P_{1}^{1, C_{1}} \neq 0$, and $m^{2}$ is the dimension of $C_{1}$. The fact that $P_{1}^{1, C_{1}} \neq 0$ implies the existence of no less than $2 p$ subspaces $P_{1}^{g^{i}, C_{i+1}}$, $P_{1}^{S\left(C_{i+1}\right), g^{p-i}}$, each of which has the same dimension as $P_{1}^{1, C_{1}}$ by Lemma 3.5. Moreover, each $P_{1}^{g^{i}}, C_{i+1} \neq 0$ implies the existence of a grouplike element $h_{i}$ such that $P_{n}^{g^{i}}, h_{i}(n \geq$ $2)$ is non-degenerate, by applying Lemma 3.8. And each $P_{1}^{S\left(C_{i+1}\right), g^{p-i}} \neq 0$ implies the existence of an $m^{2}$ dimensional simple subcoalgebra $\bar{C}_{i}$ such that $P_{n^{\prime}}^{S\left(C_{i}\right), \bar{C}_{i}}\left(n^{\prime} \geq 2\right)$ is non-degenerate by Lemma 3.8 again.

On the other hand, non-degeneracy of $P_{n}^{g^{i}, h_{i}}(n \geq 2)$ implies $P_{1}^{g^{i}, X}$ and $P_{n-1}^{X, h_{i}}$ are non-degenerate by Lemma 3.2. Since $\operatorname{dim} X \neq 1$, we can assume $X=C_{i+1}$ without loss of generality.

We consider the case $\operatorname{dim} C_{1}=4$. By the result of Lemma 4.1, there exists a positive integer $k$ such that $S\left(C_{k}\right) \notin\left\{C_{1}, \ldots, C_{p}\right\}, P_{1}^{S\left(C_{k}\right), g^{p-k+1}} \neq 0$. Set $g^{k-1} S\left(C_{k}\right)=$ $D_{1}, D_{j}=g^{j-1} D_{1}$ for fixed $k$ above. Then $\left\{C_{1}, \ldots, C_{p}\right\} \cap\left\{D_{1}, \ldots, D_{p}\right\}=0$ and $P_{1}^{D_{i}, g^{i-1}}$, $P_{n-1}^{h_{i}^{\prime}, D_{i}}, P_{n^{\prime}}^{D_{i}, \bar{D}_{i}}$ are non-degenerate where $h_{i}^{\prime}=g^{i+k-2} h_{k-1}^{-1}, \bar{D}_{i}=g^{i+k-2} \bar{C}_{k}$. Moreover, $P_{n^{\prime \prime}}^{C_{i} \overline{\bar{C}}_{i}}\left(n^{\prime \prime} \geq 2\right)$ is non-degenerate for each $i \in\{1, \ldots, p\}$ where $\overline{\bar{C}}_{i}$ is a simple coalgebra of dimension 4, since $P_{n-1}^{C_{i+1}, h_{i}}$ is non-degenerate and using Lemma 3.8. As a result of the discussion above, disjoint non-degenerate subspaces

$$
P_{1}^{g^{i}, C_{i+1}}, P_{1}^{D_{i}, g^{i-1}}, P_{n-1}^{C_{i+1}, h_{i}}, P_{n-1}^{h_{i}^{\prime}, D_{i}}, P_{n}^{g^{i}, h_{i}}, P_{n^{\prime \prime}}^{C_{i} \overline{\bar{C}}_{i}}, P_{n^{\prime}}^{D_{i} \bar{D}_{i}}\left(n, n^{\prime}, n^{\prime \prime} \geq 2\right)
$$

exist where $g^{i}, h_{i}, h_{i}^{\prime}$ are grouplikes, $C_{i}, D_{i}, \bar{D}_{i}, \overline{\bar{C}}_{i}$ are 4 dimensional simple coalgebras.

Therefore we obtain the following. If $(m, p)=1$ then $\operatorname{dim}^{1} I^{C_{1}} \geq 2 p m+p+m^{2} p$, and if $m=k p$ then $\operatorname{dim}^{1} I^{C_{1}} \geq 2 p^{2} k+p+k^{2} p^{2}$. Moreover, $\operatorname{dim}{ }^{1} I^{C_{1}} \geq(4 p \cdot 2)+(p+$ $p \cdot 4+p \cdot 4)=17 p$ for the case $\operatorname{dim} C_{1}=4$.

We set ${ }^{X} H^{Y}=\sum_{i, j}\left(g^{i} S^{j}(X)+g^{i} S^{j}(Y)\right) \oplus \oplus^{X} I^{Y}$.

If $(m, p)=1$ then ${ }^{1} H^{C_{1}}$ contains disjoint $C_{1}, \ldots, C_{p}$. Moreover, if $m=2$ then ${ }^{1} H^{C_{1}}$ contains disjoint $C_{1}, \ldots, C_{p}, D_{1}, \ldots, D_{p}$. Thus we obtain the following.

LEMMA 4.2. Let $C_{1}$ be a simple subcoalgebra such that $P_{1}^{1, C_{1}} \neq 0$, and $m^{2}$ be the dimension of $C_{1}$. If $m=2$ then $\operatorname{dim}^{1} H^{C_{1}} \geq 26 p$, and if $(m, p)=1$ then $\operatorname{dim}^{1} H^{C_{1}} \geq$ $2\left(m^{2}+m+1\right) p$, and if $m=k p$ then $\operatorname{dim}^{1} H^{C_{1}} \geq 2\left(k^{2}+k\right) p^{2}+2 p$.

COROLLARY 4.3. The dimension of any simple subcoalgebra of $\mathrm{H}$ is 1 or 4 or 9 or $p^{2}$. Moreover the following holds for the coradical $\mathrm{H}_{0}$ of $\mathrm{H}$. 
If $\operatorname{dim} C_{1}=4$ then $H_{0}=\mathbf{k C}_{p} \oplus C_{1} \oplus \cdots \oplus C_{p} \oplus D_{1} \oplus \cdots \oplus D_{p}$.

If $\operatorname{dim} C_{1}=9,(p \neq 3)$ then $H_{0}=\mathbf{k} \mathbf{C}_{p} \oplus C_{1} \oplus \cdots \oplus C_{p}$.

If $\operatorname{dim} C_{1}=p^{2}$ then $H_{0}$ is isomorphic to one of the following,

(i) $\mathbf{k C}_{p} \oplus C_{1} \quad$ (ii) $\mathbf{k} \mathbf{C}_{p} \oplus C_{1} \oplus \bar{C} \quad$ (iii) $\quad \mathbf{k C}_{p} \oplus C_{1} \oplus \bar{C} \oplus \tilde{C}$

(iv) $\quad \mathbf{k C}_{p} \oplus C_{1} \oplus B_{1} \oplus \cdots \oplus B_{p} \quad$ (v) $\quad \mathbf{k C}_{p} \oplus C_{1} \oplus \bar{C} \oplus B_{1} \oplus \cdots \oplus B_{p}$,

where $\bar{C}, \tilde{C}$ are simple coalgebras of dimension $p^{2}, B_{1}, \ldots, B_{p}$ are simple coalgebras of dimension 4.

Proof. It is obvious by counting dimensions using Lemma 4.2.

LemMA 4.4. The space $P_{n}^{g, h}$ degenerates to $P_{2}^{g, h}$ for $n \geq 3$ where $g, h \in G(H)$.

Proof. The proof of Lemma 4.4 is by contradiction.

Suppose $P_{n}^{g, h}$ is non-degenerate for $n \geq 3$. From Lemma 3.2, there exist simple coalgebras $X_{1}, X_{2}$ such that $P_{1}^{g, X_{1}}, P_{n-1}^{X_{1}, h}, P_{n-1}^{g, X_{2}}, P_{1}^{X_{2}, h}$ are non-degenerate. Since the space $P_{n-1}^{X_{1}, h}$ is non-degenerate, applying Lemma 3.2 again, there exists a simple coalgebra $X_{3}$ such that $P_{n-2}^{X_{1}, X_{3}}, P_{1}^{X_{3}, h}$ are non-degenerate. Since $H$ has no non-trivial skew primitive elements, dimensions of $X_{1}, X_{2}$ and $X_{3}$ are greater than or equal to 4 . Then $\operatorname{dim} X_{1}=\operatorname{dim} X_{2}=\operatorname{dim} X_{3}$ by the counting dimensions with Corollary 4.3.

(i) The case $\operatorname{dim} X_{1}=4$ or 9 . Since $P_{n-2}^{X_{1}, X_{3}}$ is non-degenerate, ${ }^{g} H^{X_{1}}$ contains disjoint $p$ subspaces $\left\{P_{n-2}^{\bar{g}^{i} X_{1}, \bar{g}^{i} X_{3}}\right\}$ which have not appeared in the counting argument of the proof of Lemma 4.2 where $\bar{g}$ is a non-trivial grouplike element of $H$. Therefore $\operatorname{dim} H \geq 30 p$, contradicting $q \leq 29$.

(ii) The case $\operatorname{dim} X_{1}=p^{2}$. Since $P_{1}^{g, X_{1}}, P_{n-1}^{X_{1}, h}, P_{n-1}^{g, X_{2}}, P_{1}^{X_{2}, h}, P_{n-2}^{X_{1}, X_{3}}$ are nondegenerate, ${ }^{g} H^{X_{1}}$ contains disjoint $4 p+1$ subspaces

$$
\left\{P_{1}^{\bar{g}^{i} g, \bar{g}^{i} X_{1}}\right\},\left\{P_{n-1}^{\bar{g}^{i} X_{1}, \bar{g}^{i} h}\right\},\left\{P_{n-1}^{\bar{g}^{i} g, \bar{g}^{i} X_{2}}\right\},\left\{P_{1}^{\bar{g}^{i} X_{2}, \bar{g}^{i} h}\right\}, P_{n-2}^{X_{1}, X_{3}}
$$

where $\bar{g}$ is a non-trivial grouplike element of $H$. Therefore $\operatorname{dim} H \geq 7 p^{2}+2 p$, contradicting $q<4 p+10$.

Let $C_{1}$ be a simple coalgebra such that $P_{1}^{1, C_{1}} \neq 0$. We suppose that $\operatorname{dim} C_{1}=4$ or $\operatorname{dim} C_{1}=9, p \neq 3$. Then Corollary 4.3 and Lemma 4.4 imply that there is no subspace which have not appeared in the proof of Lemma 4.2. Thus $H={ }^{1} H^{C_{1}}$ and so the dimension of ${ }^{1} H^{C_{1}}$ can be expressed as $26 p+4 z_{1}+6 z_{2}+9 z_{3}$ with positive integers $z_{i}$, by Lemma 4.4. This contradicts the condition $q \leq 29$. Therefore $\operatorname{dim} C_{1}=p^{2}$. And thus the dimension of $H$ can be expressed as $4 p^{2}+2 p+p^{2} z_{1}+4 p z_{2}$ with positive integers $z_{1}, z_{2}$.

This completes the proof of Theorem 1.1.

\section{REFERENCES}

1. N. Andruskiewitsch and S. Natale, Counting arguments for Hopf algebras of low dimension, Tsukuba J. Math. 25 (2001), No. 1, 187-201.

2. M. Beattie and S. Dăscălescu, Hopf algebras of dimension 14, J. London Math. Soc. (2) 69 (2004), No. 1, 65-78.

3. S. Dăscălescu, C. Năstăsescu and Ş. Raianu, Hopf algebras: an introduction, Monographs in Pure and Applied Math. 235 (Marcel Dekker, 2000).

4. P. Etingof and S. Gelaki, Semisimple Hopf algebras of dimension $p q$ are trivial, J. Algebra 210 (1998), No. 2, 664-669.

5. P. Etingof and S. Gelaki, On Hopf algebras of dimension pq, J. Algebra 277 (2004), No. 2, 668-674. 
6. S. Montgomery, Hopf algebras and their actions on rings, CBMS, Vol. 82 (AMS, 1993).

7. S.-H. Ng, Non-semisimple Hopf algebras of dimension $p^{2}$, J. Algebra 255 (2002), No. 1, $182-197$.

8. S.-H. Ng, Hopf algebras of dimension pq, J. Algebra 276 (2004), No. 1, 399-406.

9. S.-H. Ng, Hopf algebras of dimension 2p, Proc. Amer. Math. Soc. 133 (2005), 2237-2242.

10. D. Ştefan, Hopf subalgebras of pointed Hopf algebras and applications, Proc. Amer. Math. Soc. 125 (1997), No. 11, 3191-3193. 